\title{
The Nature of Time
}

\author{
Sydney Baldwin Self ${ }^{1}$
}

1214 Lone Laurel Trail, Hendersonville, NC 28792, USA

Correspondence: Sydney Baldwin Self, 214 Lone Laurel Trail, Hendersonville, NC 28792, USA. E-mail: sydself@alum.mit.edu

Received: December 24, 2016

doi:10.5539/apr.v9n2p21
Accepted: January 22, 2017

Online Published: February 16, 2017

URL: https://doi.org/10.5539/apr.v9n2p21

\section{Abstract}

I. Special relativity does not address the fact that time must exist on lifeless worlds. It only addresses time that is observed, which requires that observers be present.

II. The time that exists on lifeless worlds can be termed physical time.

III. The failure to recognize the existence of physical time has resulted in a view of time that is contrived, overly simplistic and contains irrational conclusions which are not experimentally supported.

IV. On worlds where conscious life exists, both physical time and observed time exist. The characteristics of physical time and of observed time are very different.

V. Physical time has the following attributes:

a. Each physical event is associated with physical time.

b. The attributes of absolute time, 'now' and time dilation are associated with every physical event.

c. All physical events occur during 'now'.

d. A physical event results in a change to physical reality.

e. The frame of reference associated with a physical event is universal.

f. In order to be observed, a physical event must have an associated observed event.

VI. Observed time has the following attributes.

a. Every observed event is associated with observed time.

b. An observed event can only occur as the result of a physical event.

c. Multiple observations can be associated with a single physical event.

d. The attribute of 'now' and a frame of reference are associated with every observed event.

VII. The duration of physical 'now' is a Planck time which is also the unit of measure for absolute time and time dilation.

VIII. Time dilation is computed using the Lorentz transformation.

IX. The statement regarding time dilation "When two observers are in relative uniform motion and uninfluenced by any gravitational mass, the point of view of each will be that the other's (moving) clock is ticking at a slower rate than the local clock" is mathematically, experimentally and logically wrong.

$\mathrm{X}$. During physical 'now' a particle may move through space or it may move through time; which it does is a probability based on the speed of the particle expressed as a percentage of the speed of light.

Keywords: Time, Special Relativity, Time Dilation, Now, Absolute Time, Duration of Now 


\section{Introduction}

In a previous article - A Logical Examination of the Nature of Time (Self, 2016), which included a discussion of special relativity, I made the point that special relativity is completely oriented to discussing time as it is perceived by observers. Special relativity does not address the fact that time must exist on lifeless worlds where there are no observers. As far as special relativity is concerned, one might conclude that time does not exist on such worlds. So much for the tree that falls in the forest!

Time in some form must exist on such worlds and, since Korzybski in his book "Science and Sanity" (Korzybski, 1994), pointed out that 'the map is not the territory' it seems likely that the nature of time on a lifeless world (where there is only territory, no maps) is not the same as the time that we perceive in our world, (where we have maps); therefore, in this article, I define such aspects of time as physical time.

Physical time must have existed on our earth before life came into being and there is no logical reason to believe that it did not continue to exist after events started being observed. Physical time must have continued to exist but it would have been augmented by those observations; in other words, the time that we experience today must include both physical time and observed time.

The characteristics of physical reality — which was what Korzybski was referring to - are quite different from those of observed reality - logically, this fact should also true of the characteristics of physical time and observed time. At present we confuse the characteristics of physical time with those of observed time; and this article will show that our failure to recognize the existence of physical time, has resulted in a view of time that is contrived, overly simplistic and contains irrational conclusions which are not experimentally supported.
Newton's view of time is similar to what I consider to be physical time, Einstein's view of time is what
I consider to be observed time. I believe that both views can and do exist, which results in a very different way of thinking about time.

\section{Time}

What is time? The simplest definition of which I am aware defines time as the tool we use to measure the duration of an event; but exactly what constitutes an event? Ohanian and Markert (Ohanian \& Markert, 2007) define an event as "an occurrence that happens at one point of space at one point of time", but I view that definition as an over simplification. Examining possible types of events, it is possible that there could be four:

- One type is the physical event. Without the existence of a physical event, there is no event to be observed!

- There are observable physical events; which may or may not actually be observed. A physical event which is not observed has no associated, observed event. A physical event which is observed generates a second kind of event; an observed event. It can be observed by one or more observers and has one or more associated, observed events.

- A third kind of event is a physical event which is, by its nature, unobservable. Unobservable physical events cannot have an associated observed event.

- The fourth kind of event is an imaginary event - it is originated as the result of a mental process; perhaps such an event can actually occur, or perhaps it can't. If it turns out that an imaginary event really can occur, then it could generate a physical event which might be observable but until its occurrence can be demonstrated it certainly can't be observed.

From the perspective of understanding the nature of time, it is important to know whether an event can, or cannot, be observed. This article will be based on the following assumptions:

- For observable events, it will be assumed that the event is observed; - even though only a small fraction of physical events are really observed - thus it will have both a physical event and an associated observed event. (For the sake of simplicity it will be assumed that, unless stated otherwise, there is only one observer).

- An event that is not observable cannot result in an associated observed event, just the physical event.

- Imaginary events will be identified as such.

\section{Observed Time}

An observed event has the following characteristics:

- An observed event can only occur as the result of a physical event.

- The characteristics of the physical event and its associated observed event(s) are not the same. 
- Each observed event must have one or more observers associated with it.

- An observed event exists in the mind of the observer, and can be documented and shared with other observers. In and of itself it leaves no record except in the mind of the observer. If more than one observer is present, each has his own personal observation of the event; such multiple observations need not be consistent with one another!

- Each observed event has a time - observed 'now' — associated with it. If there is more than one observer, there can be more than one 'observed now'. The 'now' of an observed event may occur after the 'now' of the associated physical event but it may not occur before.

\section{Frames of Reference}

All physical events have characteristics that are not observable; the only frame of reference that can be applicable to a physical event is a generic frame of reference that includes the entire universe.

Each observer of an event has a specific frame of reference that applies to that event and the observer is able to imagine the existence of frames of reference other than his own; thus there is no limit to the number of frames of reference that can exist in observed time.

The boundaries of a specific frame of reference require definition - the boundaries can include the entire universe; in which case the conditions existing in that frame of reference apply to the entire universe - or the boundaries can be limited, in which case the conditions existing in the frame of reference do not apply to those parts of the universe that are not within that frame of reference.

Frames of reference can overlap; in such cases boundaries need to be defined and considered.

\section{Physical Time}

Every physical event that happens, happens in physical time during physical 'now'. A physical event modifies physical reality, therefore it leaves a physical record of what it has done. (If it doesn't leave a physical record, it isn't an event).

Some of the attributes of physical events can be observed some can't. Two examples of attributes which cannot be observed are absolute time and time dilation. We cannot directly perceive either (although we can experience their effects).

It is difficult to talk definitively about physical time because there is very little experimental evidence which deals with it.

\section{Michelson-Morley Experiment}

Prior to the Michelson-Morley experiment (Michelson \& Morley, 1887), in the late 19th century, physicists believed that a luminiferous æther permeated space, thereby providing a medium through which light could travel.

This experiment proved that the direction which light took when it moved through space did not affect its measured speed, thereby invalidating the concept of a luminiferous æther and also changing a defining characteristic of light from vector to scalar. This discovery resulted in the concept of special relativity originated by Albert Einstein.

The Michelson-Morley experiment didn't explain how the speed of light could be a constant in all inertial reference frames, including reference frames that were travelling through space at different velocities. This difficulty was resolved by the Lorentz transformation.

\section{The Speed of Light}

To understand physical time one has to first understand the nature of the speed of light.

1) The speed of light has an absolute value. This value does not depend on the speed through space of the source of the light, the speed through space of an observer of that light source, or the direction the light is travelling.

2) According to Max Planck (CODATA Value: Planck Time, n.d.), the speed of light equals a 'Planck length' divided by a 'Planck time'. This is a very interesting statement that has not been given the attention it deserves. To begin with, it defines the speed of light not the velocity of light — the fact that 'speed' is a scalar quantity, not a vector, and does not incorporate the concept of direction is important!

3) A 'Planck length' is defined in meters; A 'Planck time' is defined in seconds; both are absolute, universal constants defined by CODATA. 
4) A Planck time constitutes the duration of physical 'now'. (Proof that 'now' exists, both in physical time and observed time, is given below.) Planck time is referred to, in this article, as a tick.

5) A Planck time is the unit of measure of absolute time. Since photons were created at the time of the big bang and some have been travelling through space ever since at the speed of light, the number of Planck times experienced by such a photon constitutes absolute time.

6) The duration of a Planck time is much too small to allow for its measurement. This is probably the reason why the importance of the concept of 'Planck time' has never been given the attention it deserves.

7) As defined by the Lorentz transformation, time dilation is a function of the speed of light; therefore time dilation must be an aspect of physical time, not of observed time.

8) Since we know that earth is moving through space and that the speed of an object through space cannot be known, it can be assumed that all objects that exist on earth are moving through space, thus, time dilation is a characteristic that applies to all earthly objects.

9) Photons travel at the speed of light, thus, as proven by the Lorentz transformation, they experience $100 \%$ time dilation. Objects that are not moving through space (if any exist) experience no time dilation.

\section{The Nature of 'Now'}

The first question regarding 'now' that must be addressed is "Does 'now' exist"?

Einstein claimed that 'now' didn't exist! One of the major points made in my referenced article relates to the existence of 'now'. In that article I invoked Ockham's razor by asking a second question 'If 'now' doesn't exist, when does anything happen?" In my article I proposed that not only does 'now' exist, but that it must exist. Let us consider my reasoning.

To begin, "Is 'now' an aspect of physical time or is it an aspect of observed time?" To answer that question, I'll use an example.

Suppose we have a pile driver which is operating at night so that it is not visible but is known to make a stroke every second. There is an accurate clock associated with the pile driver which records the exact time of each stroke. It is important to recognize that each stroke of the pile driver constitutes a physical event and the time at which it occurs defines the physical 'now' for that event.

Consider the nature of the physical 'now' associated with the pile driver. Each stroke of the pile driver drives the pile deeper into the ground - each is a physical event. Each stroke generates sound - creating a sound wave. The creation of each sound wave is also a physical event. Each of these events is a unique event which occurs at a specified, identifiable time, termed here as physical 'now'. These events (both the stroke itself and the creation of the associated sound wave) are aspects of physical reality.

Although the strokes of the pile driver are not seen, they can be heard by nearby observers, each of whom has an accurate clock, (the clocks are all synchronized). It is important to note that the time shown on each observers' clock is a running record of the 'observed now' of that observer.

The observers are situated at random locations which are at different distances from the pile driver, but since it is dark, the observers do not know where the pile driver is located and, in particular, they do not know how far they are from the driver; therefore they have no way of knowing how long the sound takes to travel from the pile driver to their location.

When each observer hears the sound of a stroke by the pile driver, each records the exact time, as shown on his clock. A key point here is that since the observers are at different distances from the pile driver, that 'observed now' will be different for each observer and, not only are the times recorded different, but the different observers are not necessarily recording the time each hears as associated with the same stroke of the pile driver!

To explain, starting with the first stroke of the pile driver, assuming that the speed of sound is 340 meters per second, when the observers who are 340 meters, or less, from the pile driver hear the sound made by the first stroke of the pile driver and their clocks will all read one second, or less, than those who are more than 340 meters distant but less distant than 680 meters will not be hearing the sound actually made by the pile driver for that first stroke until their clocks read more than one second. This is because it took the sound an additional second to travel the extra distance, and each additional 340 meters requires an additional second. As the pile driver makes more strokes, the observers who are further away will always be recording a later time than the ones who are closest, but the records for each observer are not, as they are recorded, associated with a particular stroke. 
Next, let us consider what the observers observe. When a sound wave is generated by the stroke of a pile driver and then observed by an observer, it constitutes a observed event for that particular observer. But other than making a minor change within the brain of the observer, no change to physical reality occurs. Each observer records a observed 'now' for each stroke of the pile driver. Since each observation is unique; there are many observed 'now's for each stroke of the pile driver - one for each observer - , each recorded at a different time, but these observations are not aspects of physical reality, they are aspects of observed reality!

If one attempts to sequence these observations, there is a problem; there is no easy logical way to do it; the observations are not necessarily in sequence by the number of the stroke, nor are they in sequence by observer - the observed 'now' by observer \#2 of stroke \#2 could precede the observation by observer \#1 of stroke \#1 (because observer \#1 could be further from the pile driver than observer \#2).

Thus the answer to the question asked above — "Does 'now' exist" — is that Einstein did not recognize that there were two aspects of time which had different characteristics; he didn't distinguish between the characteristics of physical time and those of observed time. In other words, the answer to the question asked above is that both observed 'now' and physical 'now' exist, but they have different characteristics; physical 'now' is a unique, absolute, constant which 'ticks' continuously and is synchronized throughout the universe. Observed 'now' is a characteristic associated with an individual observer, so it is not unique. That was Einstein's problem with 'now' - since each observer had his own 'now', and since physical 'now' wasn't recognized, there was no way to reach agreement as to whose 'now' was the one when the event actually took place! Furthermore, observed 'now' was not defined by Planck, so it is not a constant and it is certainly not an absolute. (When one is driving a car down a highway the observed 'now's flow into one another - by the time you say 'now', the past has already arrived).

The above establishes the fact that 'now' exists, both as physical 'now' and observed 'now', but what are its characteristics?

- When is 'now'?

- What is the duration of 'now'?

To answer these questions in order:

- Physical 'now' is when all physical events happen.

- Observed 'now' is the 'now' experienced by observers - each observer has his own 'now'.

- The duration of a physical event is measured in ticks. Each tick must be a constant; if it weren't physical 'now' could not be synchronized. An event may (and usually does) require more than one tick - equal to a Planck time - (if it were not, the speed of light would not have the value it does).

- The duration of observed 'now' is not a constant and has no defined value.

- Physical 'now' is now throughout the entire universe, and it is synchronized. Application of Occam's razor shows that 'now' must be synchronized; if physical 'now' were not synchronized the universe would be completely chaotic.

- Observed 'now' is not synchronized.

\section{Absolute Time}

One of the characteristics of physical time is absolute time. Time ticks, and ticks continuously, one 'now' at a time; and has been ticking at that rate since the big bang (or perhaps longer). It defines the duration of all physical events.

According to Newton, absolute time exists independently of any observer and progresses at a consistent pace throughout the universe. He believed that absolute time was imperceptible and could only be understood mathematically by measuring the movement of objects through space. (I see no reason to contradict Newton on this definition).

But since, as defined above, absolute time is not based on physical measurements, then there is no longer a reason to reject absolute time on those grounds.

Special relativity rejects absolute time for an entirely different reason - as discussed above, special relativity does not distinguish between observed time and physical time. It also does not recognize that there are aspects of time that cannot be observed - namely time dilation and absolute time. (To me that seems like throwing the baby out with the bath water!) 
However, when Planck established (and defined) the concepts of Planck length and Planck time - the speed of light equals a Planck length (1.616 x $10^{-35}$ meters) divided by a Planck time $\left(5.39 \times 10^{-44}\right.$ seconds $)$ - he, in effect, defined them as absolutes. Both have dimensions which are too short to be measured, but logically both must exist.

As further proof, around the time of the big bang, there must have been many, many photons generated. Astronomers today still receive some of these photons; they have been travelling from the location of the big bang (wherever that was) to the astronomer's telescope ever since. We can't measure the duration of these trips but we can accept them, as a concept, as an illustration of the existence of absolute time.

Logically, the never ending, sequential passage of the ticks of Planck time meets the definition of 'absolute time'. Therefore, I propose that the concept of absolute time be re-established using the number of ticks it takes for a photon to move from one location to another as the base for determining a useful value for absolute time. (Joseph Larmor can stop turning in his grave)!

It will be shown as part of the discussion of the Lorentz transformation that the definition of time dilation assumes the existence of absolute time.

\section{The Lorentz Transformation (Lorentz, 1904)}

Hendrik Lorentz, Joseph Larmor, and others were instrumental in developing what is known as the "Lorentz transformation", which defines the computation of the 'Lorentz factor'. The formulation of the Lorentz transformation was done concurrently with Einstein's development of the theory of special relativity. Larmor is given credit for being the first to understand a crucial characteristic of physical time, namely time dilation.

The Lorentz transformation defines how to calculate time dilation, which is the reduction of the speed of an object which results from the speed of an object through space - the greater the speed, as it relates to the speed of light, the greater the time dilation.

When one tries to measure the time dilation of the Earth, apparently Earth moves so slowly in relation to the speed of light that there is no detectable time dilation - this explains why it had not been detected earlier.

The Lorentz transformation defines the speed of light as a scalar quantity, not a vector. (This is an important characteristic that is not always recognized.)

The equation for computing the Lorentz factor is:

$$
\gamma=\frac{1}{\sqrt{1-\frac{v^{2}}{c^{2}}}}
$$

As can be seen in the above equation, a basic characteristic of the Lorentz factor is that its value is based on dividing the speed of the measured object thru space by the speed of light — in other words, the Lorentz transformation is based on the relationship between the speed of an object and the speed of light; defined as a percentage.

The fact that space contains no base point from which the speed of an object can be measured has been known for a very long time and it is an interesting point that the equation for the Lorentz factor neatly avoids this problem by allowing the speed of an object be related to the speed of light; in other words the absolute speed of an object is based on the speed of light, which is defined as an absolute constant! Thus the result of the computation of time dilation is based on absolute time!

\section{Time Dilation}

What is time dilation?

The concept of time dilation was originally developed to explain the discovery by Michelson and Morley that the speed of light is a constant regardless of its direction. During the 1890's Hendrik Lorentz explained time dilation mathematically and developed the equation for its computation, discussed above.

So, what is time dilation? Time dilation defines the rate by which an object ages as a function of the speed that it is travelling through space, thus it relates to the speed of light. Assume a space ship travelling through space at $60 \%$ of the speed of light. According to the Lorentz transformation, an analysis of the effect of time dilation on the space ship and its contents shows that the space ship and all of its contents are aging at $80 \%$ of absolute time. The only way that the passengers on the ship can discover that time is passing more slowly for them is by getting messages from other locations, travelling at a different speed, which describe the rates that objects at their location, are aging. It is assumed that all of the aging rates are measured using the Lorentz transformation and that the time required for the message to get from one location to the other is taken into consideration. 


\section{Frisch-Smith Experiment}

In 1963, Frisch and Smith conducted experiments at different elevations in New England which allowed them to measure the time dilation of objects experiencing speed at close to the speed of light for different periods of time.

This experiment is based on the behavior of muons, which are unstable subatomic particles with a mean lifetime of $2.2 \mu \mathrm{s}$. Muons are created in large numbers as decay products resulting from collisions of cosmic rays with particles in the Earth's atmosphere (Demtröder, 2006).

If time dilation didn't exist muons would decay before they reached the surface of the earth but, as a result of time dilation, the muons age more slowly and reach the surface of the earth before they have decayed completely.

Frisch and Smith measured the decay rate of muons at these elevations because their speed had been slowed by their passage through the atmosphere thus the physical processes affecting the characteristics of the muons they measured occurred at a slower rate than did the ones travelling at their normal, much greater, speed.

The tests confirmed the predictions of the Lorentz transformation equation but although they didn't realize it, they did more than confirm the Lorentz transformation, they proved (to me at least) that the aging process generated by time dilation affects the physical age of the muons.

This statement needs explanation. All of the discussions of time dilation that I have read illustrate the discussion using rocket ships, so the observers of the experiment are part of the frame of reference that is subject to time dilation. The muon experiment is different - in the muon experiment it is the muons that are the subject; they, and only they, comprise the frame of reference. Muons react to their environment and can be affected by it; they do not possess the power to observe anything. The observers (and their clocks) were not in the same frame of reference as the muons. The 'clock' used to measure the time dilation of the muons was the duration of the decay rate of the muons themselves. Muon decay is a physical process whose duration is measured by the physical process itself, but it was also measured by the observers clock which was running at the normal speed of other clocks on earth. Furthermore, the time measured by the physical process could not have been observed time, it could only have been absolute time.

When I first learned of time dilation, which was prior to my learning of the experiments done by Frisch and Smith, I had not recognized that special relativity did not distinguish between perceived time and physical time. As a result I was puzzled by trying to understand how a particle could 'know how old it was'! If a particle carried with it no record of how old it was, how could (as stated by Brian Greene in his "The Fabric of the Universe" (Green, 2004)) “. . . each of us carry our own yardstick"? The Frisch and Smith experiment caused me to recognize the fact that particles did not need to "carry a yardstick of how old [a person] is" the yardstick itself provided the fact that the age of the observer who experienced time dilation was actually less than the age of an observer who had not!

It was the recognition of the nature of this event that was the source of my conclusion that time dilation affected physical time, not observed time.

A point that is rarely mentioned is that the earth is moving through space, (although its speed through space is not known); so every object on earth, including all devices for measuring time such as clocks, moves through space at the same rate as earth, and is affected by time dilation. This includes the clocks used by Frisch and Smith. This time dilation is probably very close to zero so it is not observable and is never taken into consideration by experiments addressing time dilation, but there may be galaxies which are travelling through space much faster than we are and the creatures (if any) on these galaxies might experience time much more slowly than we do.

It is relevant that we use formulae, such as the Lorentz transformation, that can illustrate and explain time dilation mathematically, but no one has explained exactly why time moves more slowly for particles that are travelling at a high speed than it does for particles moving more slowly. I believe that I have an explanation!

In my article "A Logical Explanation of the Nature of Time", I formulated the theory that during physical 'now' a particle may move through space, or it may move through time. Which it 'does is based on its speed expressed as a percentage of the speed of light. If the particle moves through space it moves a distance of a Planck length but does not age (this is the source of time dilation). If it moves through time it ages by a Planck time but does not move through space; it may not do both during the same tick; thus, speed can be thought of as a probability. 


\section{Special Relativity}

Special relativity contains anomalies. In my opinion, the most serious one is the lack of recognition that time consists of both observed time and physical time. I consider it to be the most serious one because, if I am correct, many - if not all - of the precepts of special relativity will have to be re-written and there will be a lot of painful re-learning that will be necessary. But then, special relativity itself required a lot of re-learning.

This defect also results in some subordinate anomalies. One is the fact that special relativity rejects the concept of 'now' because of the conflict between physical 'now' and observed 'now'.

Another is the rejection of the concept of absolute time. Absolute time is useful in itself - astronomers depend on it - but it is also the base used to compute time dilation.

A considerably less important anomaly is that the second postulate of special relativity states that 'The speed of light in a vacuum is the same for all observers ...' This postulate would seem to limit itself so that it only applies to observed time; this cannot be correct.

Almost as important as the lack of distinction between physical time and observed time is the misunderstanding of the nature of time dilation which shows up in a number of references. I'll quote the description of time dilation in Wikipedia which states "When two observers are in relative uniform motion and uninfluenced by any gravitational mass, the point of view of each will be that the other's (moving) clock is ticking at a slower rate than the local clock." Paul Davies in his book "About Time" (Davies, 1995) says "Suppose we have two observers A and B, each with a synchronized clock ... moving relative to each other. From A's reference frame, it is clock B that is moving, and hence slowed by time dilation. But in B's reference frame, it is A that is moving and therefore running slow." Ohanian and Markert in Physics for Engineers and Scientists, Ian Stewart in "Flatterland", Max Born in "Einstein's Theory of Relativity, and Taylor \& Wheeler in Spacetime Physics all say essentially the same thing. This anomaly is recognized in many places

The 'twins paradox' is also concerned with the same problem. Supposedly, it has been resolved by proposing a fiddly approach to the turnaround of one of the twins, but logical analysis of that solution shows that it is not relevant.

How can I be correct and these reputable sources be wrong? I have three reasons:

1) These sources are mathematically wrong. The Lorentz transformation equation, which defines the computation of time dilation, requires that the speed of the object under consideration be divided by the speed of light. The calculations used by these sources ignores that requirement.

2) There is no experimental evidence to support these sources; not the Michelson-Morley experiment, not the Frisch-Smith experiment, not the Hafele-Keating experiment. In the Frisch-Smith experiment, if one were to use the conventional approach and compute the relative time dilation between two different muons (assuming it was possible to measure the relative speed of individual muons), the time dilation experienced by a muon would depend on whether you compared its speed to another muon or to the time dilation experienced by the observer.

3) It is obvious that the existing method of calculation would not give a logical answer. Also, in the Frisch-Smith experiment, a muon moving at high speed does not have the option of changing its frame of reference. Individual muons cannot have individual frames of reference; they all must use the universal frame of reference.

4) These sources are logically wrong, if there were no other evidence the twins paradox should be evidence enough, but there are more logical proofs. To use Davies' example for the purposes of illustration, let us put some numbers on the speeds of the two observers. Let us assume that observer $\mathrm{A}$ is on a space station which is traveling through space at the same speed as Earth; observer B is on a rocket ship traveling at $80 \%$ of the speed of light. Using the Lorentz transformation, observer B is aging at a rate that is $60 \%$ of the aging of observer A, who is aging at the rate of other observers on Earth.

Changing the reference point - saying that each observer views the speed of the other as affected by time dilation - is the equivalent of saying that if $\mathrm{A}$ is sitting on an oak tree and $\mathrm{B}$ is in a car traveling past it at 40 miles an hour, that it is ok to suddenly reverse the speeds and say that the car is stationary and the oak tree is moving at 40 miles an hour! In fact, reversing the reference points is the equivalent of saying that it is ok to think of B as stationary and the whole universe as being in motion at $80 \%$ of the speed of light!

Recognizing that the Lorentz transformation requires that each speed be divided by the speed of light does not change the speed at which either observer is moving through space, but it does eliminate the apparent 
paradox. In other words, the problem lies either in a misunderstanding of the Lorentz transformation by special relativity or that the Lorentz transformation is wrong! (I'll stand by the Lorentz transformation).

In my article "A Logical Examination of the Nature of Time" I modify the experiment by having A and B be pregnant twins, one of whom travels. When the twins end their trip their babies, who were impregnated at the same time, are not the same physical age anymore and, changing the reference point, even if it were possible, couldn't change the age of the babies!

But let us modify the experiment a bit more, let us introduce observer $\mathrm{C}$, also with a clock and let observer $\mathrm{C}$ also be traveling at $80 \%$ of the speed of light but in exactly the opposite direction (in reference to $\mathrm{A}$ ) as observer B. The absolute speeds of both observer B and observer C in reference to A's frame of reference are the same - remember that the Lorentz transformation defines speed as a scalar quantity so the time dilation and aging of observer B and of observer C are the same. But observer B and observer C are physically moving away from each other at a rate of $160 \%$ of the speed of light. (This does not conflict with Einstein he only talks about the comparative speed of an object and the speed of light). But how can the time dilation in reference to $\mathrm{B}$ and $\mathrm{C}$ be calculated directly? The Lorentz transformation fails if one tries to apply it to the combined vectors of the two observers, it could only be applied to each one separately.

But let us modify the experiment again; after a certain period of time, $\mathrm{B}$ and $\mathrm{C}$ both reverse their direction and return to their original location, again traveling at the same speed. As they travel, their frames of reference to A are the same as before as are their time dilations and aging, but now, according to existing theory, the time dilation of each, as it relates to the other, is zero. This is not logically acceptable and the reason is that time dilation is an aspect of physical reality not of observed reality. The relative velocity between objects has no meaning in physical reality, only in observed reality.

To summarize, when using the Lorentz transformation, if the speeds of two or more objects are to be compared, the time dilation of each must be separately computed, in physical time, using the Lorentz transformation and then, and only then, can the results be compared! Calculating the relative speed difference and then applying the Lorentz transformation is not consistent with physical reality. The problem with the conventional approach is that it confuses observed time with physical time.

\section{References}

CODATA Value: Planck Time. (n.d.). In The NIST Reference on Constants, Units, and Uncertainty.

Davies, P. (1995). About Time. Orion Productions (p. 59).

Demtröder, W. (2006). Experimentalphysik (4th ed.). Springer.

Green, B. (2004). The fabric of the cosmos. NY: Vintage Books Inc.

Korzybski, A. (1994). Science and sanity: An introduction to non-Aristotelian systems and general semantics (5th ed). Institute of GS.

Lorentz, H. A. (1904). Electromagnetic phenomena in a system moving with any velocity smaller than that of light. In KNAW Proceedings (Vol. 6, pp. 1903-1904).

Michelson, A. A., \& Morley, E. W. (1887). On the Relative Motion of the Earth and of the Luminiferous Ether. American Journal of Science, 34, 333-345.

Ohanian, H. C., \& Markert, J. T. (2007). In Wiegman L. A. W. (Ed.), Physics for engineers and Scientists (Extended Third ed.). New York: W. W. Norton \& Company Inc.

Self, S. B. (2016). A logical examination of the nature of time. Physics Essays, 29(2), 261-271.

\section{Copyrights}

Copyright for this article is retained by the author(s), with first publication rights granted to the journal.

This is an open-access article distributed under the terms and conditions of the Creative Commons Attribution license (http://creativecommons.org/licenses/by/4.0/). 